\title{
Forsterite amorphisation by ion irradiation: Monitoring by infrared spectroscopy
}

\author{
J. R. Brucato ${ }^{1}$, G. Strazzulla ${ }^{2}$, G. Baratta ${ }^{2}$, and L. Colangeli ${ }^{1}$ \\ 1 INAF Osservatorio Astronomico di Capodimonte, via Moiariello 16, 80131 Napoli, Italy \\ e-mail: colangeli@na. astro.it \\ 2 INAF Osservatorio Astrofisico di Catania, via S.Sofia 78, 95123 Catania, Italy \\ e-mail: gianni@ct.astro.it, gbaratta@ct.astro.it
}

Received 20 May 2003 / Accepted 15 September 2003

\begin{abstract}
We present experimental results on the crystal-amorphous transition of forsterite $\left(\mathrm{Mg}_{2} \mathrm{SiO}_{4}\right)$ silicate under ion irradiation. The aim of this work is to study the structural evolution of one of the most abundant crystalline silicates observed in space driven by ion irradiation. To this aim, forsterite films have been synthesised in the laboratory and irradiated with low energy (30-60 keV) ion beams. Structural changes during irradiation with $\mathrm{H}^{+}, \mathrm{He}^{+}, \mathrm{C}^{+}$, and $\mathrm{Ar}^{++}$have been observed and monitored by infrared spectroscopy. The fraction of crystalline forsterite converted into the amorphous form is a function of the energy deposited by nuclear collision by ions in the target.

Laboratory results indicate that ion irradiation is a mechanism potentially active in space for the amorphisation of silicates. Physical properties obtained in this work can be used to model the evolution of silicate grains during their life cycle from evolved stars, through different interstellar environments and up to being incorporated in Solar System objects.
\end{abstract}

Key words. methods: laboratory - techniques: spectroscopic - ISM: dust, evolution - ISM: cosmic rays

\section{Introduction}

During late stages of evolution, stars lose mass in a short time compared to the time they spend in the main sequence. In the gaseous expanding circumstellar envelopes, dust condensation occurs. In particular, the envelopes of giant stars belonging to the asymptotic branch (AGB) (Gehrz 1989) and to supernovae (SN) (Jones 1997) are considered the principal sites of cosmic dust formation. Once formed, grains are injected in the interstellar medium (ISM) driven by the stellar winds.

Recent detailed observations of AGB M-type stars in the medium and far infrared spectral regions obtained with the ISO satellite revealed series of bands testifying that both amorphous and crystalline silicate phases are present in the envelope (Kemper et al. 2001; Molster et al. 2002a,b,c). The results showed the presence of two principal crystalline silicate components, forsterite $\left(\mathrm{Mg}_{2} \mathrm{SiO}_{4}\right)$ and enstatite $\left(\mathrm{MgSiO}_{3}\right)$. Relevant correlations between silicate grain properties and outflow or disk source geometries were found. In particular, disklike stars show strong crystalline silicate bands, while outflow geometry is characterised by weak crystalline silicate bands. Amorphous silicates were detected at temperatures higher than those of crystalline ones. Molster et al. (2002c) showed that enstatite and forsterite masses correlate with each other and

Send offprint requests to: J. R. Brucato,

e-mail: brucato@na.astro.it that enstatite is more abundant than forsterite by a factor of 3-4. Fitting analysis by laboratory spectra of the Spectral Energy Distributions (SED) indicates that, for example, in the source $\mathrm{OH}-127.8+0.0,80 \%$ of silicates are amorphous olivine $\left((\mathrm{Mg}, \mathrm{Fe})_{2} \mathrm{SiO}_{4}\right), 3 \%$ are forsterite, $3 \%$ enstatite, $4 \%$ metallic iron, and $10 \%$ crystalline water ice (Kemper et al. 2002). A value of $3 \%$ for forsterite and enstatite was also calculated for many AGB stars (Sylvester et al. 1999) and considered typical for these stars (Kemper et al. 2001).

The distribution of crystalline and amorphous silicate grains according to AGB mass-loss rates was derived (Sogawa \& Kozasa 1999; Kemper et al. 2001; Suh 2002). In particular, Kemper et al. (2001) showed that the presence of crystalline silicates does not depend on the mass-loss rate. Moreover, they demonstrated that the lack of spectroscopic signatures of crystalline silicates in the ISO spectra does not imply the absence of crystalline dust in the envelope. This result was obtained considering that, if two separate populations of amorphous and crystalline grains are used in the radiative transfer model of circumstellar envelopes, up to $40 \%$ of crystals can be included in the envelope dust without observing any spectroscopic evidence.

Further analyses of AGB stars spectra were performed by Suh (2002) by using a different dust model. An average single grain population for mixed amorphous and crystalline silicate grains was chosen. It was obtained that about 10 to $20 \%$ of 
crystalline silicates are produced in high mass-loss rate AGB stars, but no crystalline silicates were found in low mass-loss rate stars. The discrepancy in these results depends on the formation mechanism chosen to be active in AGB envelopes. In the first case, two grain populations are formed with different temperatures, while in the second case a single amorphous component is formed that subsequently crystallises totally or partially by annealing, reaching a single final temperature.

The model of Sogawa \& Kozasa (1999) already confirmed the absence of crystalline silicates in heterogeneous grains condensed in low mass-loss stars $\left(\leq 3 \times 10^{-5} M_{\odot} \mathrm{yr}^{-1}\right)$. However, condensation models of minerals in stellar winds of M-stars indicate that a multicomponent mixture is mainly formed, dominated by olivine and iron grains (Gail \& Sedlmayr 1999). The temperature evolution of the condensed grains in the outflow favours the birth of crystalline grains when their radius is $\leq 100 \mathrm{~nm}$. For larger grains the temperature is too low to crystallise the materials, giving rise to a further population of crystalline core grains with an external coating of amorphous material.

Thus, if we consider that AGB stars with circumstellar envelopes are expected to be ubiquitous in our Galaxy (Habing 1996) and that they account for $50 \%$ of the total stellar massloss of the Galaxy, the crystalline silicate component formed in these stars should be observed in the Interstellar medium (ISM). On the contrary, analysis of ISO spectra showed that an upper limit of only a few percent of crystals is present in dense and diffuse ISM (Li \& Draine 2001; Demyk et al. 1999).

Among various mechanisms that could explain the absence or the non-observability of crystalline silicates in the ISM (e.g. selective destruction, low production rate or interstellar dust dilution) in this work we experimentally investigate the amorphisation process by interaction with $30-60 \mathrm{keV}$ ions.

Previous laboratory irradiation experiments of forsterite with energetic (1.5 MeV) protons (Day 1977) and of clinoenstatite with $400 \mathrm{keV}$ and $1 \mathrm{MeV}$ helium ions (Jäger et al. 2003), made at fluences similar to those of cosmic rays, did not show any infrared spectroscopic evidence of structural modification of crystals. Complete amorphisation for low energy ( 4 and $10 \mathrm{keV}$ ) helium ions irradiation at similar fluences was observed (Demyk et al. 2001; Carrez et al. 2002).

The crystal-amorphous transition process can be affected significantly by various physical parameters of the impinging ions, as e.g. the mass, charge and the kinetic energy. Thus, further laboratory studies are needed to investigate the amorphisation process of silicates.

In this work, experimental results on structural modifications suffered by crystalline forsterite under ion irradiation are presented. Thin films of forsterite are synthesised in order to monitor in situ, by infrared transmission spectroscopy, the crystalline to amorphous transition during irradiation. Processing effects are investigated, the correlation with the energy deposition is studied and the cross-section of nuclear collision which is directly related to amorphisation is derived. In Sect. 2 we describe the experimental apparatus and procedures. Results are presented in Sect. 3 and discussed in Sect. 4. Astrophysical implications of experimental results and concluding remarks are reported in Sect. 5.

\section{Experimental}

Among various laboratory techniques that give information on the chemical and physical properties of silicates of various nature, infrared spectroscopy is widely used. The results obtained by this technique can be used directly for comparison with space observations, once a dust model is applied. A series of infrared spectra are available in the literature for different classes of crystalline silicates where peak positions and intensities were linked to properties of the samples, such as morphology, chemical composition and crystallographic structure (e.g. Koike et al. 1993; Mennella et al. 1998; Jäger et al. 1998; Koike et al. 2000; Fabian et al. 2001; Suto et al. 2002; Chihara et al. 2002; Brucato et al. 2002; Colangeli et al. 2003). In the present work the experimental procedure used is based on the possibility to acquire transmission infrared spectra of crystalline silicate samples and to observe their evolution during the irradiation process. To this aim, thin films of crystalline forsterite were prepared in laboratory for in situ infrared transmission spectroscopy and ion bombardment processing.

\subsection{Thin silicate film synthesis}

Silicate films were prepared at the Cosmic Physics Laboratory of INAF-Osservatorio Astronomico di Capodimonte by using a Nd-YAG solid state pulsed laser. Its fundamental wavelength output is at $1064 \mathrm{~nm}$ with a mean energy of $650 \mathrm{~mJ}$. The power laser output is $10^{8} \mathrm{~W} \mathrm{~cm}^{-2}$ per laser pulse. A set of two crystals was used to get II and IV harmonics at 532 and $266 \mathrm{~nm}$, respectively. The energy output was $80 \mathrm{~mJ}$ at $266 \mathrm{~nm}, 120 \mathrm{~mJ}$ at $532 \mathrm{~nm}$ and $190 \mathrm{~mJ}$ at $1064 \mathrm{~nm}$. By an optical set-up, the $266 \mathrm{~nm}$ wavelength was selected and directed onto the target. By using a focusing lens, the power density was maintained at $10^{8} \mathrm{~W} \mathrm{~cm}^{-2}$ by compensating for the decreased mean energy. Targets were prepared by using mixtures of periclase $(\mathrm{MgO})$ and quartz $\left(\mathrm{SiO}_{2}\right)$ at the stochiometric ratio $(2: 1)$ of forsterite $\mathrm{Mg}_{2} \mathrm{SiO}_{4}$. The mixture was pressed at 10 tons obtaining pellets $13 \mathrm{~mm}$ in diameter and a few millimetres thick. The target pellet was mounted inside a vaporisation chamber which was designed to be filled by different gases. The presence of a quenching reactive gas affects the chemical composition of the laser ablated sample, while its pressure drives the morphology of the condensed materials. The lower the pressure inside the chamber the smaller the size of the condensed grains. In previous laser ablation experiments the vaporisation chamber was filled with oxygen at 10 mbar (Brucato et al. 1999, 2002). This was done to maintain the chemical composition of the condensed sample similar to that of the target. Moreover, the presence of oxygen favours the super-saturation of the hot quenched vapour. This is responsible for the formation of grains with sizes following a log normal distribution with average size of few tens of nanometers. In order to produce films thin enough to obtain observable transmission IR spectra before and during ion irradiation, the chamber pressure was maintained at $10^{-5}$ mbar. With these experimental conditions the mean free path of the atoms of the plasma plume produced by the laser ablation can be considered infinitely larger than the distance target-sample $(3 \mathrm{~cm})$. Thus, the super saturation condition, 


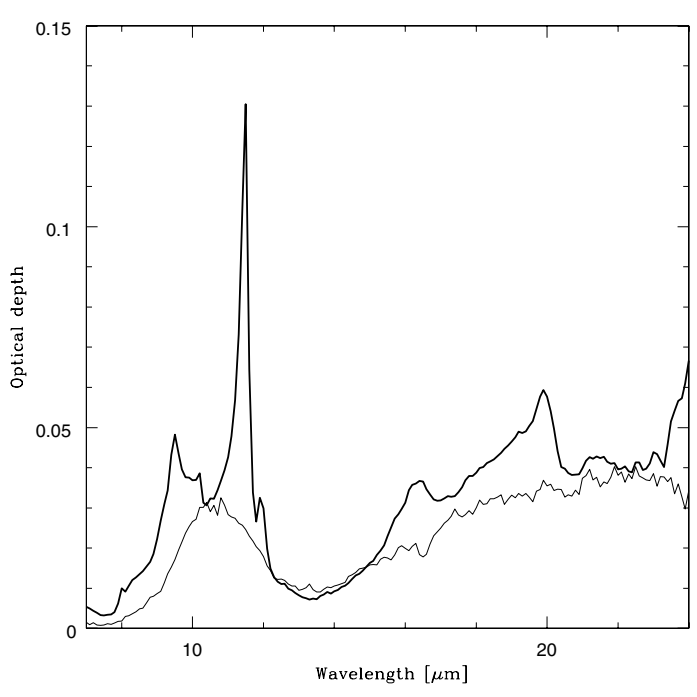

Fig. 1. Infrared optical depth spectra of laser synthesised amorphous film (thin line) and of crystalline forsterite after thermal annealing (thick line).

necessary to condense grains, is not reached along the path of the atoms to the substrate. Films of amorphous silicates are produced after 15 min deposition on silicon wafer substrates. The infrared spectrum of the condensed sample, shown in Fig. 1, presents two smooth and broad bands at 10.6 and around $20 \mu \mathrm{m}$ typical of amorphous silicate.

In order to prepare samples with crystalline structure, the amorphous silicate films were annealed at $900{ }^{\circ} \mathrm{C}$ for 1 hour at the pressure of $10^{-6}$ mbar. The annealing temperature is reached at a rate of $23{ }^{\circ} \mathrm{C} \mathrm{min}{ }^{-1}$ and controlled electronically. The transition from the amorphous to crystalline phase is seen by the appearance in the infrared spectum of a series of sharp bands (Fig. 1), typical of crystalline materials.

\subsection{Ion irradiation}

Ion irradiation was performed by a Danphysik (1080-30) ion implantation system of the INAF-Astrophysical Observatory laboratory of Catania. The gas is ionised in the source and a reduced pressure favours the production of a plasma. Ions are accelerated by a $30 \mathrm{kV}$ potential and mean kinetic energies of $30 \mathrm{keV}$ and $60 \mathrm{keV}$ are obtained for singly and double ionised ions, respectively. Once extracted, the ions travel through a mass separator where defined $\mathrm{m} / \mathrm{q}$ are selected by a magnetic field. An electrostatic scanning system is used to deflect the ion trajectories. This allows us to irradiate uniformly the sample with low current density (of the order of $1 \mu \mathrm{A} \mathrm{cm}^{-2}$ ), avoiding undesirable annealing of the sample. The target assembly is designed for in situ spectroscopy. For further details on the experimental apparatus see Strazzulla et al. (2001). In the present experiment, samples were irradiated with $30 \mathrm{keV} \mathrm{H}{ }^{+}, \mathrm{He}^{+}, \mathrm{C}^{+}$ and with $60 \mathrm{keV} \mathrm{Ar}{ }^{++}$with ion fluences up to $10^{17} \mathrm{~cm}^{-2}$. Light (hydrogen and helium) and heavy (carbon and argon) ions were chosen to study how different classes of ions affect silicate structure.

The thin films were characterised by Fourier transform infrared spectroscopy (Bruker Equinox 55) in the mid-infrared

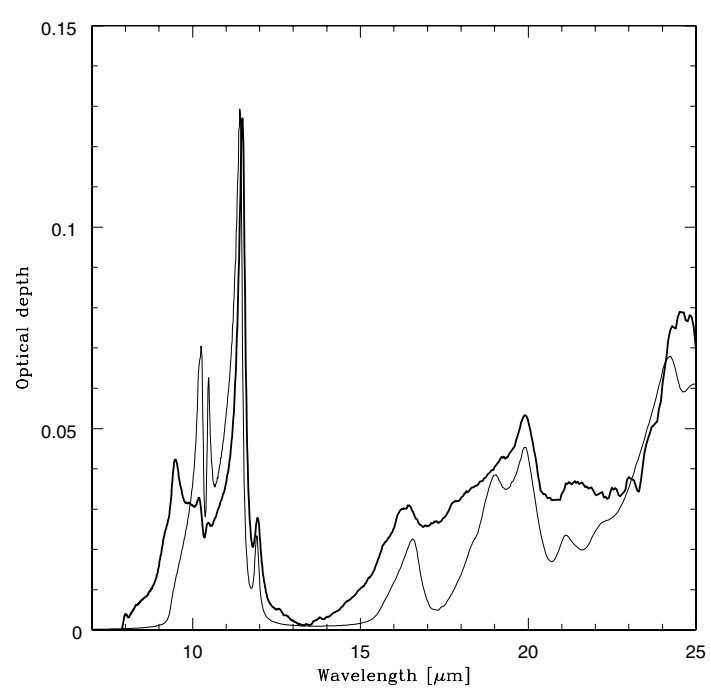

Fig. 2. Infrared optical depth spectra of films of synthesised forsterite normalised to the continuum (thick line) and of crystalline olivine $\left(\mathrm{Mg}_{1.9} \mathrm{Fe}_{0.1} \mathrm{SiO}_{4}\right)$ calculated by the optical constant of Fabian et al. (2000) (thin line).

with resolution of $4 \mathrm{~cm}^{-1}$. Spectra were acquired after laser deposition and thermal annealing and in situ during ion irradiation of samples, in order to monitor the chemical and physical evolution of the silicate films.

\section{Results}

\subsection{Chemical composition and thickness}

In order to check composition and thickness, the IR spectrum of synthesised films was compared to that of crystalline olivine. The optical constants of $\mathrm{Mg}_{1.9} \mathrm{Fe}_{0.1} \mathrm{SiO}_{4}$ olivine derived by Fabian et al. (2000) were used to evaluate the optical depth, $\tau$, of the film. This is defined according to the equation $\tau=4 \pi d k / \lambda$, where $d$ is the thickness of the film and $k$ is the imaginary part of the complex refractive index. The average absorbance $\tau=\frac{1}{3}\left[\tau_{x}+\tau_{y}+\tau_{z}\right]$ along the $(x, y, z)$ crystallographic axes for a film of thickness $d=380 \AA$ is reported in Fig. 2 and compared with the absorbance of a thin film sample synthesised in this work. Unfortunately, the sample used by Fabian et al. is not the pure forsterite $\left(\mathrm{Mg}_{2} \mathrm{SiO}_{4}\right)$ end member of olivines, a small fraction of iron being present. The presence of iron in olivine induces a shift in the peak positions towards longer wavelengths that according to Jäger et al. (1998) is correlated to the mass percentage of $\mathrm{FeO}$ according to the relation: $[\mathrm{FeO}] / \Delta v=-1.8 \pm 0.1$.

Comparing the spectra in Fig. 2 the presence of all the peaks of forsterite in the synthetic sample spectrum is evident, even if a mismatch of peak positions and intensities is observed. Differences in peak intensities are probably due to a preferential axis of growth of the crystals during the thermal annealing. Peak positions are reported in Table 1, together with the peak positions expected for a pure forsterite thin film after correction of wavelength shifts. The peak at $9.48 \mu \mathrm{m}$ is due to the presence of a fraction of quartz which does not participate in olivine formation. 
Table 1. Peak positions for synthetic forsterite film, olivine $\mathrm{Mg}_{1.9} \mathrm{Fe}_{0.1} \mathrm{SiO}_{4}$ film as obtained by optical constant from Fabian et al. (2000), and after wavelength shift correction for pure forsterite according to Jäger et al. (1998).

\begin{tabular}{ccc}
\hline \hline Forsterite & Olivine & $\begin{array}{c}\text { Forsterite } \\
\text { corrected }\end{array}$ \\
\hline 9.48 & - & - \\
10.19 & 10.25 & 10.28 \\
10.46 & 10.48 & 10.51 \\
11.50 & 11.42 & 11.46 \\
11.94 & 11.92 & 11.96 \\
16.31 & 16.56 & 16.64 \\
- & 19.02 & 19.12 \\
19.91 & 19.91 & 20.02 \\
21.35 & 21.11 & 21.24 \\
- & 22.18 & 22.32 \\
24.5 & 24.21 & 24.38 \\
\hline
\end{tabular}

Table 2. Nuclear, $S_{\mathrm{n}}$, and electron, $S_{\mathrm{e}}$, stopping powers and penetration ranges, $R$, in the forsterite target computed by the TRIM Montecarlo simulation program for ions with kinetic energy, $E$.

\begin{tabular}{ccccc}
\hline \hline Ion & $E[\mathrm{keV}]$ & $S_{\mathrm{n}}[\mathrm{eV} / \AA]$ & $S_{\mathrm{e}}[\mathrm{eV} / \AA]$ & $R[\AA]$ \\
\hline $\mathrm{H}^{+}$ & 30 & 0.08 & 16 & 2300 \\
& 1500 & 0.003 & 5.0 & $1.9 \times 10^{5}$ \\
$\mathrm{He}^{+}$ & 4 & 2.5 & 3.6 & 324 \\
& 10 & 1.7 & 7.4 & 800 \\
& 30 & 0.9 & 16 & 2000 \\
$\mathrm{C}^{+}$ & 30 & 12.5 & 27 & 630 \\
$\mathrm{Ar}^{++}$ & 60 & 86 & 43 & 431 \\
\hline
\end{tabular}

In order to give a further estimate of the film thickness, analysis of scanning electron micrographs of the samples was performed. The samples attached onto aluminium stubs were tilted by about 90 degrees to show the side. The thickness of film portions showing sharp edges was measured and an average film thickness of $500 \pm 200 \AA$ was obtained. This value is compatible with that obtained by the calculation of the optical depth and comparable with the penetration ranges of the ions (Table 2).

\subsection{Ion amorphisation}

Infrared spectra of forsterite irradiated with $30 \mathrm{keV} \mathrm{He}^{+}$at different fluences are shown in Fig. 3. A progressive decrease of the intensities of the crystalline peaks is observed when increasing the ion fluence and the two large and smooth bands at around 10 and $20 \mu \mathrm{m}$, typical of the amorphous silicates, appear in the spectra. A similar trend is observed in Fig. 4, where spectra of forsterite irradiated with $30 \mathrm{keV} \mathrm{H}^{+}$and $\mathrm{C}^{+}$ are shown. A residual crystalline fraction is still present in the irradiated samples, as is shown by the low intensity residue crystalline peaks observed in Fig. 3 for the sample irradiated at the highest fluence. This fluence is that for which an extended ion irradiation does not produce further observable variations in peak intensities. This means that the samples, at the

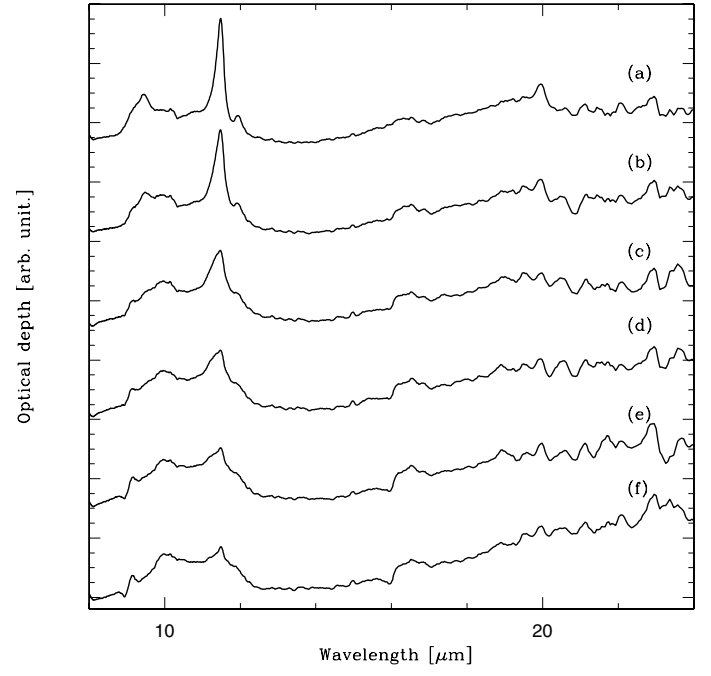

Fig. 3. Evolution of infrared optical depth spectra of forsterite (a) before and after irradiation with (b) $8.0 \times 10^{14}$, (c) $4.9 \times 10^{15}$, (d) $1.0 \times$ $10^{16}$, (e) $2.4 \times 10^{16}$, and (f) $1.04 \times 10^{17} \mathrm{He}^{+} \mathrm{cm}^{-2}$ with kinetic energy of $30 \mathrm{keV}$.

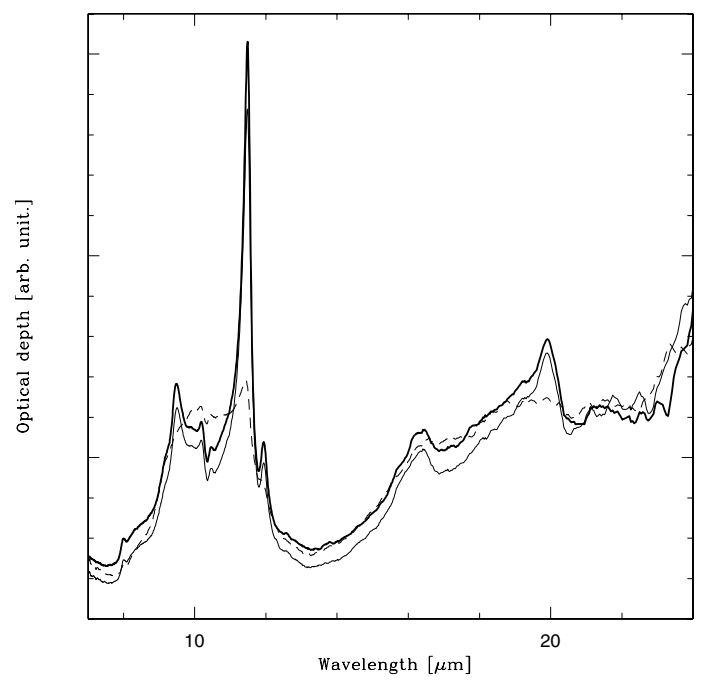

Fig. 4. Evolution of infrared optical depth spectra of forsterite before (thick line) and after irradiation of $6.8 \times 10^{15} \mathrm{H}^{+} \mathrm{cm}^{-2}$ (thin line), and of $1.4 \times 10^{15} \mathrm{C}^{+} \mathrm{cm}^{-2}$ (dashed line) with kinetic energies of $30 \mathrm{keV}$. The crystal damage induced by proton irradiation is negligible if compared to that due to carbon ions, even if the kinetic energies are the same and the fluence of proton irradiation is about 5 times larger then that of carbon ions.

end of the process, are not completely amorphisised. Moreover, ejecta of micron sizes coming from the laser target were observed in scanning electron micrographs to be deposited with the film (Fig. 5). The inclination of 45 degree of the target surface with respect to the ion beam of the implanter device could prevent complete ion processing of a fraction of the film which is shielded by the ejecta. Since the film thicknesses are of the order of or smaller than the ion penetration ranges, complete amorphisation of the film occurs. The residual crystalline fraction can be associated with the shielding by the ejecta grains of the film below. Moreover, the ejecta could be forsterite grains with sizes larger than the ion penetration range, that are not 


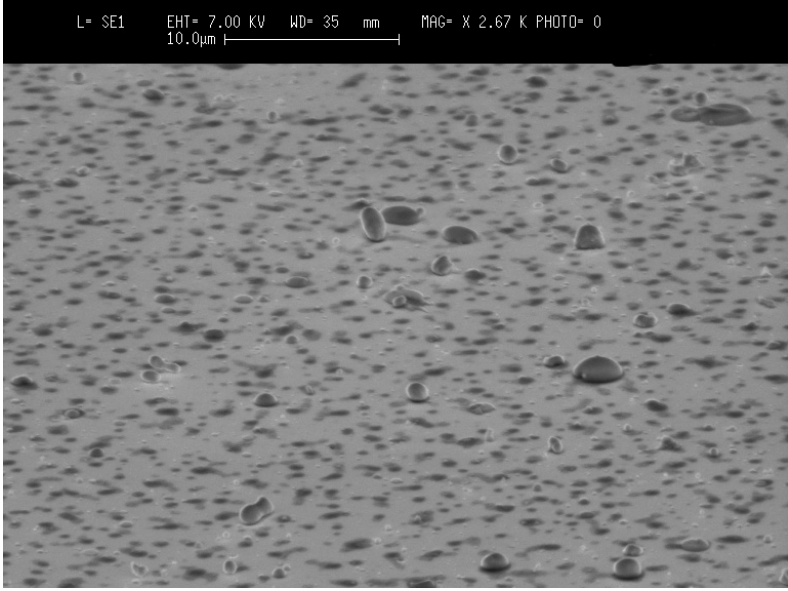

Fig. 5. Scanning electron micrograph of forsterite film. Ejecta coming from the laser target are observed with the silicate film.

completely amorphised by the ion irradiation, which does not produce observable infrared spectra in transmittance.

\section{Discussion}

The spectra in Figs. 3 and 4 show that, for about the same ion fluence, the irradiation of forsterite with different ions at the same kinetic energy produces different amounts of damage of the crystalline structure. This is shown by different intensities of sharp (crystalline) and broad (amorphous) bands. In order to quantify the effects on the crystal-amorphous phase transition of forsterite, the infrared spectra, $S$, are fitted by a linear combination of crystalline, $S_{\mathrm{c}}$, and amorphous, $S_{\mathrm{a}}$, spectra of forsterite (Fig. 1):

$S=F_{\mathrm{c}} \cdot S_{\mathrm{c}}+F_{\mathrm{a}} \cdot S_{\mathrm{a}}$

where $F_{\mathrm{c}}$ and $F_{\mathrm{a}}$ are the fractions of crystalline and amorphous components, respectively.

It is also important to evaluate the energy deposition rate on forsterite versus mass, charge, and kinetic energy of the ions. The mean energies deposited by the impinging ions, through nuclear (elastic), $S_{\mathrm{n}}$, and electronic (anelastic), $S_{\mathrm{e}}$, collisions with the target atoms along the ions path were computed by the TRIM Montecarlo simulation program and are reported in Table 2. To determine whether the amorphisation process depends on the energy deposition process in the target, the fractions of crystalline forsterite, $F_{\mathrm{c}}$, remaining after the irradiation versus nuclear, $D_{\mathrm{n}}=\Phi \cdot S_{\mathrm{n}}$, and electronic, $D_{\mathrm{e}}=\Phi \cdot S_{\mathrm{e}}$, irradiation doses are considered, where $\Phi$ is the ion fluence. The results are plotted in Fig. 6.

Similar experiments were performed by irradiating forsterite grains with $1.5 \mathrm{MeV}$ of $\mathrm{H}^{+}$(Day 1977) and with 4 and $10 \mathrm{keV}$ of $\mathrm{He}^{+}$(Demyk et al. 2001). The results can be compared with our data in terms of $F_{\mathrm{c}}$ vs. $D_{\mathrm{n}}$. No change in the infrared spectra of $\mathrm{H}^{+}$irradiated forsterite was observed by Day (1977) $\left(F_{\mathrm{c}}=1\right)$, while a complete amorphisation was observed by transmission electron microscopy by Demyk et al. (2001) after irradiation with $\mathrm{He}^{+}\left(F_{\mathrm{c}}=0\right)$. Starting from the fluences reported by the authors, $7 \times 10^{17} 1.5 \mathrm{MeV} \mathrm{H}^{+} / \mathrm{cm}^{2}$ (Day 1977) and $5 \times 10^{16} 4 \mathrm{keV} \mathrm{He} / \mathrm{cm}^{2}$ and $10^{18} 10 \mathrm{keV} \mathrm{He}^{+} / \mathrm{cm}^{2}$
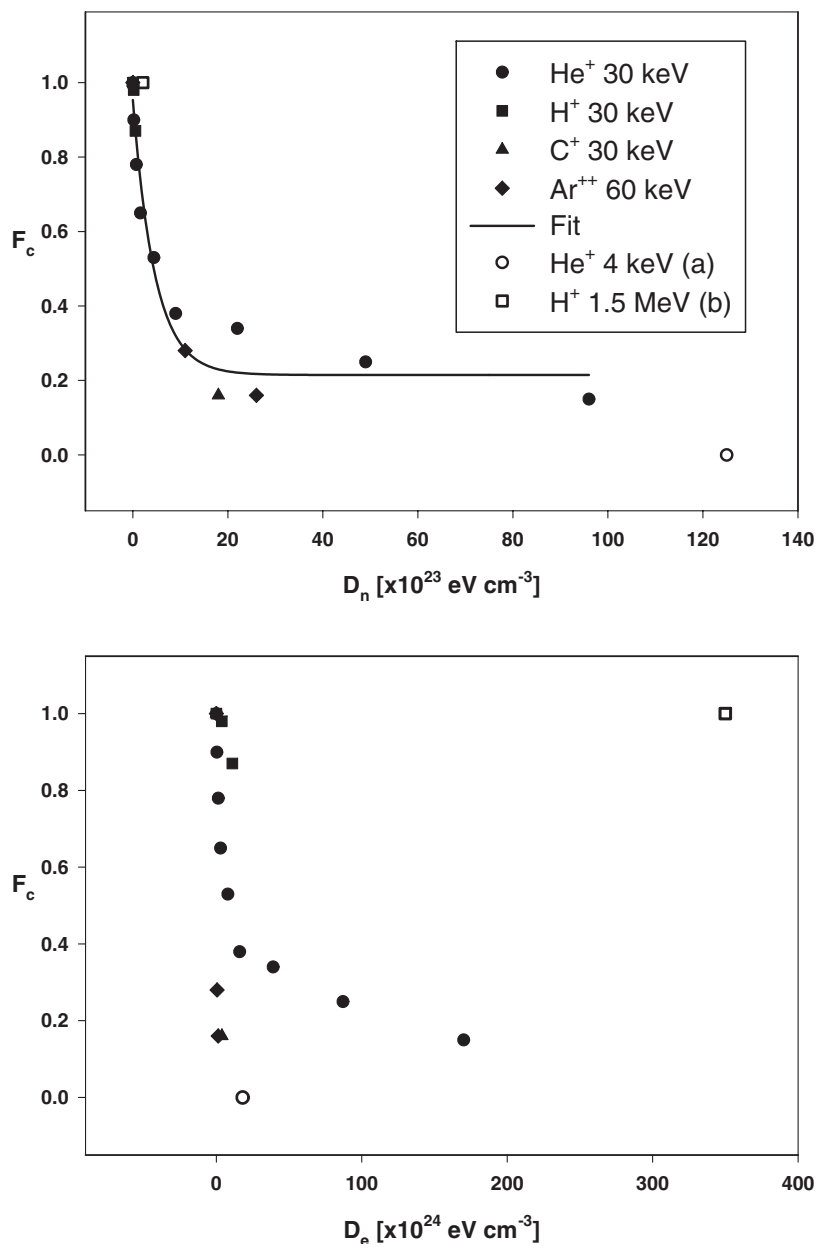

Fig. 6. Top panel: the evolution of forsterite crystalline fraction, $F_{\mathrm{c}}$, versus nuclear (elastic) doses, $D_{\mathrm{n}}$. The thin line represents the fit by a decreasing exponential law $F_{\mathrm{c}}=0.21+0.74 \cdot \exp \left(-2.2 \times 10^{-24} \cdot D_{\mathrm{n}}\right)$ to the evolution of the crystalline fraction of forsterite, $F_{\mathrm{c}}$, versus nuclear dose, $D_{\mathrm{n}}$, for different ion irradiations. Bottom panel: the evolution of forsterite crystalline fraction, $F_{\mathrm{c}}$, versus electronic (anelastic) doses, $D_{\mathrm{e}}$, for different ion irradiations. Values deduced from the experiments of (a) Demyk et al. (2001) and (b) Day (1977) are also reported.

(Demyk et al. 2001), $D_{\text {n }}$ are calculated by using the $S_{\text {n }}$ of Table 2. The data correlate well with those obtained in this work, if $F_{\mathrm{c}}$ is reported as a function of $D_{\mathrm{n}}$, while further missed correlation among the data is evident if $F_{\mathrm{c}}$ is reported in funcion of $D_{\mathrm{e}}$. To quantify the correlation of $F_{\mathrm{c}}$ versus $D_{\mathrm{n}}$, the function:

$F_{\mathrm{c}}=F_{\mathrm{c} 0}+A \cdot \exp \left(-\kappa \cdot D_{\mathrm{n}}\right)$

is fitted to the data points, where $F_{\mathrm{c} 0}$ is the asymptotic non irradiated crystalline fraction, $A$ is the fraction of the film that has been amorphised by the ion irradiation and $\kappa$ is the cross-section expressed in $\mathrm{cm}^{3} \mathrm{eV}^{-1}$. This quantity represents the volume of crystalline forsterite amorphised per unit energy deposited by elastic collisions. The data points describe the trend of destruction of the crystalline structure and follow well a decreasing exponential law (Fig. 6). The best fit is obtained for $F_{\mathrm{c} 0}=0.21 \pm 0.03, A=0.74 \pm 0.04$, and $\kappa=2.2 \pm 0.4 \times 10^{-24} \mathrm{~cm}^{3} \mathrm{eV}^{-1}$ with a coefficient of determination $R^{2}=0.97$. From Fig. 6 (bottom panel) it is evident that 


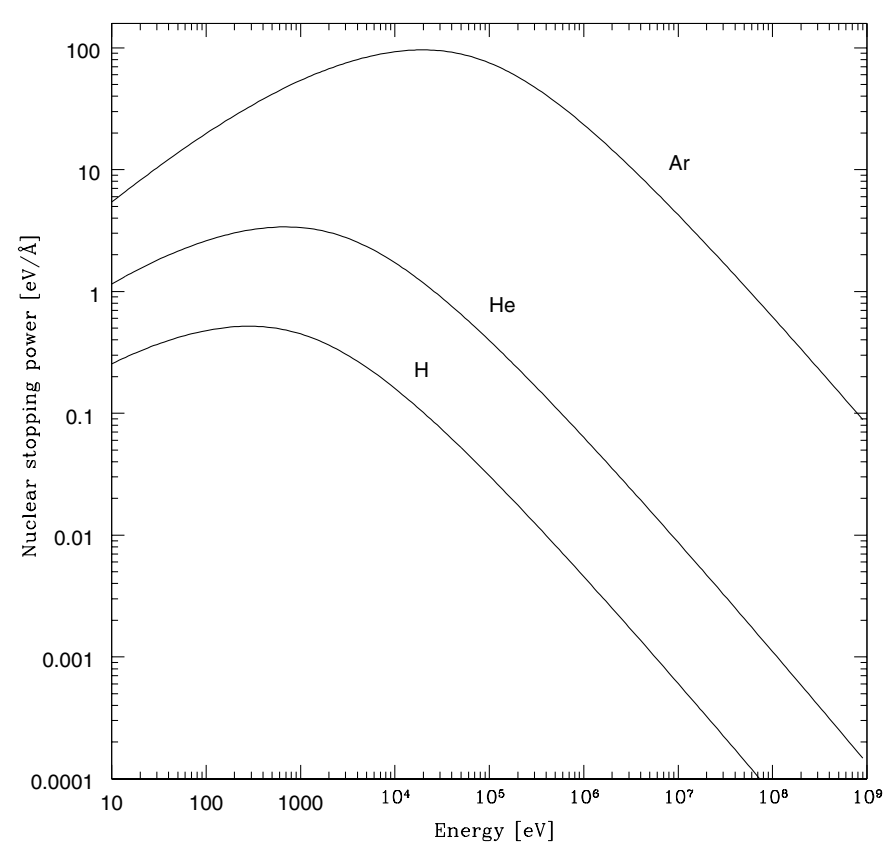

Fig. 7. Nuclear stopping power of $\mathrm{H}, \mathrm{He}$ and $\mathrm{Ar}$ ions in forsterite target versus energy, as computed by the TRIM Montecarlo simulation program.

$F_{\mathrm{c}}$ and $D_{\mathrm{e}}$ do not correlate. The dependence of the forsterite amorphisation on nuclear (elastic) dose demonstrates that it is a consequence of the effect of displacement of the target atoms by nucleus-nucleus collisions due to the impinging ions.

The results obtained in this work confirm that low energy ion irradiation is an efficient process for the amorphisation of silicate grains, strongly dependent on $D_{\mathrm{n}}$.

For forsterite, the $S_{\mathrm{n}}$ of the light $\mathrm{H}, \mathrm{He}$, and heavy Ar ions were computed by TRIM Montecarlo simulation for kinetic energies in the range of $10 \mathrm{eV}$ to $1 \mathrm{GeV}$ (Fig. 7). The stopping power increases with increasing incident ion energy, reaches a maximum and, at higher energies, decreases. The maximum stopping is obtained at higher energies as the mass of the ion increases $\left(S_{\mathrm{n}}(\mathrm{H})=0.5 \mathrm{eV} / \AA\right.$ at $0.28 \mathrm{keV} ; S_{\mathrm{n}}(\mathrm{He})=3.4 \mathrm{eV} / \AA$ at $0.7 \mathrm{keV} ; S_{\mathrm{n}}(\mathrm{Ar})=96.2 \mathrm{eV} / \AA$ at $\left.20 \mathrm{keV}\right)$. However, over the whole energy range, the stopping is larger by about 2 orders of magnitude for argon with respect to hydrogen. This means that about $1 \%$ of heavier elements produces the same structural effects as hydrogen on forsterite.

\section{Astrophysical application}

To study if ion irradiation in space is efficient to amorphise forsterite it is necessary to know the flux in space of the ions crossing the grains according to their energies. The intensity of cosmic rays is relatively well known at energies exceeding a few $\mathrm{GeV}$, but becomes increasingly uncertain at lower energies. The intensity measured on Earth understimates the lowerenergy particles which are swept back out into the interstellar space due to the presence of the solar magnetic field.

Measures of cosmic ray intensities for nuclei of various charges were made by different authors and extrapolated down to energies as low as few tens of $\mathrm{MeV}$ per nucleon. The minimum cosmic ray flux in interstellar space was evaluated by Spitzer \& Tomasko (1968) and converted to proton densities by using the relative abundances of particles given by Webber (1967). The analytical form of the lower limit of cosmic ray intensity adopted by Spitzer \& Tomasko (1968) is:

$J(E)=$

$$
\frac{0.90}{\left(0.85+E_{\mathrm{G}}\right)^{2.6}} \frac{1}{\left(1+0.01 / E_{\mathrm{G}}\right)} \text { particles } \mathrm{cm}^{-2} \mathrm{~s}^{-1} \mathrm{sterad}^{-1}
$$

where $E_{\mathrm{G}}$ is the kinetic energy in unit of $10^{9} \mathrm{eV}$. This equation is applicable in the range of kinetic energies for nucleons of $10 \mathrm{MeV}-10 \mathrm{GeV}$. Assuming that the time, $t$, spent by a grain of radius $a=0.1 \mu \mathrm{m}$ in the interstellar medium is $10^{7}-10^{8} \mathrm{yr}$, using the cross-section for the amorphisation by ion irradiation of forsterite derived in this work, the fraction of forsterite grains that is amorphised by high-energy ions can be written as $F_{\mathrm{c}}=1-\exp \left(-4 \pi^{2} a^{2} t k J(E) S_{\mathrm{n}}\right)$. For protons with energies larger then $10 \mathrm{MeV}, S_{\mathrm{n}}$ is less then $6 \times 10^{-4} \mathrm{eV} \AA^{-1}$ (Fig. 7); therefore, the amorphised fraction of the interstellar grains is negligible. Cosmic ray irradiation has no effect on the crystalline infrared features. This is true even if heavy ions induce more damage to the crystalline structure than hydrogen, according to the different nuclear stopping powers (Fig. 7).

Since $S_{\mathrm{n}}$ has its maximum at kinetic energies of the order of tens of keV (Fig. 7), the effects induced by low-energy ions which are also present in the interstellar space have to be considered. It has been calculated that supernovae generate interstellar shock waves, with velocities $V_{\mathrm{s}} \geq 50 \mathrm{~km} \mathrm{~s}^{-1}$, able to destroy by sputtering significant amount of dust present in the ISM independently of composition (Seab 1988; Mckee 1989; Jones et al. 1996). High velocity shock waves are able to propagate through large volumes of low density interstellar medium, but are reduced up to vanishing in dense molecular clouds (McKee 1989). Moreover, supernova shocks interacting with the interstellar grains may damage the crystalline structures through the mechanism studied in this work, as already indicated by Demyk et al. (2001), Carrez et al. (2002), and Jäger et al. (2003). Even if the intensity of low-energy ions as a function of their kinetic energy is not known, about $10^{18}-10^{19} \mathrm{H} \mathrm{cm}^{-2}$ are accelerated in a supernova shocks at velocities $\geq 100 \mathrm{~km} \mathrm{~s}^{-1}$ (Jones et al. 1996). These atoms impinge on dust grains with energies $\geq 52 \mathrm{eV}$ and with $D_{\mathrm{n}}=$ $4 \times 10^{25}-10^{26} \mathrm{eV} \mathrm{cm}^{-1}$. According to the data in Fig. 6, these doses are sufficient to induce a complete amorphisation of forsterite grains with a size of about $40 \AA$, with a consequent large effect on the infrared spectra. This would explain the broad band silicate spectra observed in the ISM.

The results obtained in this work and those obtained by Demyk et al. (2001), Carrez et al. (2002) and Jäger et at. (2003) demostrate that the amorphisation process due to ion irradiaton may be an efficient process in space and it is able to explain the presence of amorphous silicates in the ISM. In this work it has been shown that the amorphisation process of forsterite depends on the nuclear elastic collisions between the impinging ions and the target atoms. In contrast, no correlation has been obtained between the irradiation effects on the silicate structure and anelastic electron collisions. This result shows that the 
process of damage in the case of forsterite acts differently than on water ice, for which a dependence on the total (elastic + anelastic) dose was observed (Leto \& Baratta 2003). Large effects on the silicate infrared spectral features are produced by irradiation of low-energy ions that could be responsible for the absence of crystalline peaks in the ISO spectra of silicate dust in the ISM.

Acknowledgements. We are grateful to F. Spinella for the technical support given during the ion irradiation experiments. We would like to thank S. Inarta for his collaboration for the SEM analysis. This research has been supported by the MIUR.

\section{References}

Brucato, J. R., Colangeli, L., Mennella, V., Palumbo, P., \& Bussoletti, E. 1999, A\&A, 348, 1012

Brucato, J. R., Mennella, V., Colangeli, L., Rotundi, A., \& Palumbo, P. 2002, PSS, 50, 829

Carrez, P., Demyk, K., Cordier, P., et al. 2002, Meteor. Planet. Sci., 37,1599

Chihara, H., Koike, C., Tsuchiyama, A., Tachibana, S., \& Sakamoto, D. 2002, A\&A, 391, 267

Colangeli, L., Henning, Th., Brucato, J. R., et al. 2003, A\&ARv., 11, 97

Day, K. L. 1977, MNRAS, 178, 49

Demyk, K., Jones, A. P., Dartois, E., Cox, P., \& d'Hendecourt, L. 1999, A\&A, 394, 267

Demyk, K., Carrez, Ph., Leroux, H., et al. 2001, A\&A, 368, L38

Fabian, D., Jäger, C., Henning, Th., Dorschner, J., \& Mutschke, H. 2000, A\&A, 364, 282

Fabian, D., Posch, Th., Mutschke, H., Kerschbaum, F., \& Dorschner, J. 2001, A\&A, 373, 1125

Gail, H. P., \& Sedlmayr, E. 1999, A\&A, 347, 594
Gehrz, R. 1989, Sources of Stardust in the Galaxy. In Interstellar Dust, ed. L. J. Allamandola, \& A. G. G. M. Tielens (Dordrecht: Kluwer Academic Publishers), IAU Symp., 135, 445

Habing, H. J. 1996, A\&ARv., 7, 97

Jäger, C., Molster, F. J., Dorschner, J., et al. 1998, A\&A, 339, 904

Jäger, C., Fabian, D., Schrempel, F., et al. 2003, A\&A, 401, 57

Jones, A. P., Tielens, A. G. G. M., \& Hollenbach, D. J. 1996, ApJ, 469,740

Jones, A. P. 1997, The Lifecycle of Interstellar Dust, in From Stardust to Planetesimals, ed. Y. J. Pendleton, \& A. G. G. M. Tielens, ASP Conf. Ser., 122, 97

Kemper, F., Waters, L. B. F. M., de Koter, A., \& Tielens, A. G. G. M. 2001, A\&A, 369, 132

Kemper, F., de Koter, A., Waters, L. B. F. M., Bouwman, J., \& Tielens, A. G. G. M. 2002, A\&A, 384, 585

Koike, C., Shibai, H., \& Tuchiyama, A. 1993, MNRAS, 264, 654

Koike, C., Tsuchiyama, A., Shibai, H., et al. 2000, A\&A, 363, 1115

Leto, G., \& Baratta, G. A. 2003, A\&A, 397, 7

Li, A., \& Draine, B. T. 2001, ApJ, 550, L213

McKee, C. F. 1989, ApJ, 345, 782

Mennella, V., Brucato, J. R., Colangeli, L., et al. 1998, ApJ, 496, 1058

Molster, F. J., Waters, L. B. F. M., Tielens, A. G. G. M., \& Barlow, M. J. 2002a, A\&A, 382, 184

Molster, F. J., Waters, L. B. F. M., \& Tielens, A. G. G. M. 2002b, A\&A, 382, 222

Molster, F. J., Waters, L. B. F. M., Tielens, A. G. G. M., Koike, C., \& Chihara, H. 2002c, A\&A, 382, 241

Seab, C. G. 1988, Grain destruction and growth, in Dust in the universe (Cambridge University Press), 303

Sogawa, H., \& Kozasa, T. 1999, ApJ, 516, L33

Spitzer, L. Jr., \& Tomasko, M. G. 1968, ApJ, 152, 972

Suh, K. W. 2002, MNRAS, 332, 513

Suto, H., Koike, C., Sogawa, H., et al. 2002, A\&A, 389, 568

Sylvester, R. J., Kemper, F., Barlow, M. J., et al. 1999, A\&A, 252, 587

Strazzulla, G., Baratta, G. A., \& Palumbo, M. E. 2001, AcSpe, 57, 825

Webber, W. R. 1967, AJ, 72, 836 